\title{
Pulmonary Mucormycosis in Layer Birds: A Case Report
}

\author{
V. Samatha, B.B. Manasa1, V. Rama Devi², K. Satheesh ${ }^{3}$ \\ Department of Veterinary Pathology, \\ NTR College of Veterinary Science, Gannavaram-521 102, Andhra Pradesh, India.
}

Received: June 2021

Accepted: July 2021

\begin{abstract}
The present paper reports a case of pulmonary mucormycosis in a 19 wk layer bird that was presented for necropsy to the Department of Veterinary Pathology, NTR College of Veterinary Science, Gannavaram. Mycotic pneumonia in poultry is commonly due to Aspergillus spp and due to others like Mucor spp is very rare. On postmortem examination, the lungs were enlarged, consolidated with multiple greyish white nodules. Histologically, the lung sections revealed pyogranulomatous lesions with branched aseptate hyphae in center of necrosed area that was surrounded by heterophils, macrophages, lymphocytes and multi nucleated giant cells. These fungal hyphae were broad and a few showing right angle and/or irregular branching characteristic of Zygomyces spp. with periodic acid Schiffs reagent
\end{abstract}

Key words: Mycotic pneumonia, Periodic acid schiff staining, Zygomyces spp.

Mycosis is one of the opportunistic infections affecting mammals and birds. Aspergillosis and candidiasis are the most common mycotic infections in birds. Though Aspergillus spp. are the main causative agents for respiratory infections, few cases of zygomycosis or mucormycosis were also reported in chickens, ostriches and ducks (Perelman and Kuttin, 1992; McCaskey and Langheinrich, 1984; Migaki et al., 1970). Zygomycosis is a polymorphic disease of multiple etiology, caused by fungi of the class Zygomycetes (Chandler et al., 1980). The present paper describes the pathological findings of mycotic pneumonia in layer bird suggestive of zygomycosis.

\section{Case history}

A 19 week aged layer bird was presented for necropsy at NTR College of Veterinary Science, Gannavaram. Grossly the bird was emasciated and detailed necropsy examination revealed the lesions suggestive of Mycotic pneumonia. Lung samples were collected in $10 \%$ formalin for histopathological examination. After routine tissue processing and paraffin embedding, the lung sections were stained by haematoxylin and Eosin. Duplicate sections were subjected for PAS (Periodic Acid Schiff) staining for demonstration of fungus.

Grossly, lungs were enlarged with diffuse consolidation along with the presence of multiple greyish white nodules with diameter varying from 1 to $5 \mathrm{~mm}$ on dorsal aspect of lung surface (Fig 1). These findings were similar to the findings of a mucor mycosis affected penguin. Necropsy examination of penguin revealed multiple nodules up to 5 $\mathrm{mm}$ in diameter over the dorsal side of the lung surface (Sujuta et al., 2015).
Histologically the lung sections revealed diffuse necrotic areas characterized by pyogranulomatous inflammatory reaction. Necrotic areas surrounded by heterophils, macrophages, lymphocytes and multi nucleated giant cells along with the presence of branched aseptate hyphae. Irregular shaped hyphae with bulbous dilatation in extremities were present (Fig 2,3). These fungal hyphae were broad and a few showing right angle or irregular branching and were positively stained for PAS (Fig 4,5).

In tissues, zygomycetes are characterized by irregularly branched hyphae with broad width that may be at right angles and rarely septated. The demonstration of aseptate/ irregularly branched hyphae along with diffuse pyogranulomatous inflammation and necrosis in lung sections are suggestive of Zygomycosis. These histological

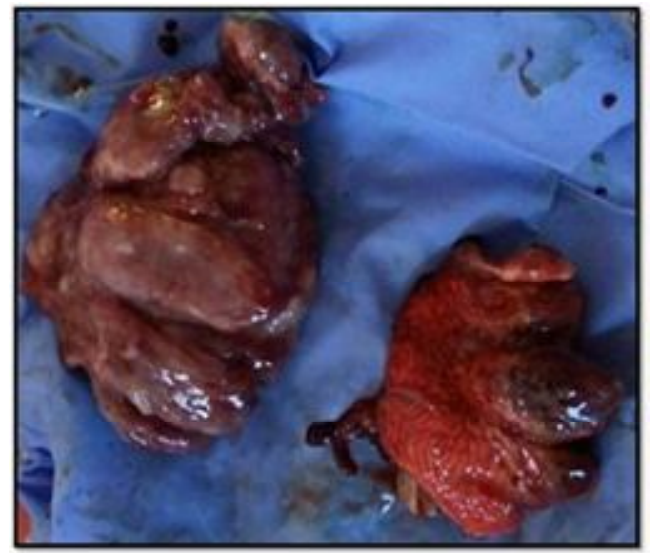

Fig 1: Enlarged lungs with presence of greyish white nodules.

*Corresponding author's E-mail: samathavety@rediffmail.com

'Veterinary Assistant Surgeon, Veterinary Biological and Research Institute, Andhra Pradesh, India.

${ }^{2}$ Department of Veterinary Pathology, NTR College of Veterinary Science, Gannavaram-521 102, Andhra Pradesh, India.

${ }^{3}$ Animal Husbandry Polytechnic, Banvasi, Kurnool-518 323, Andhra Pradesh, India. 


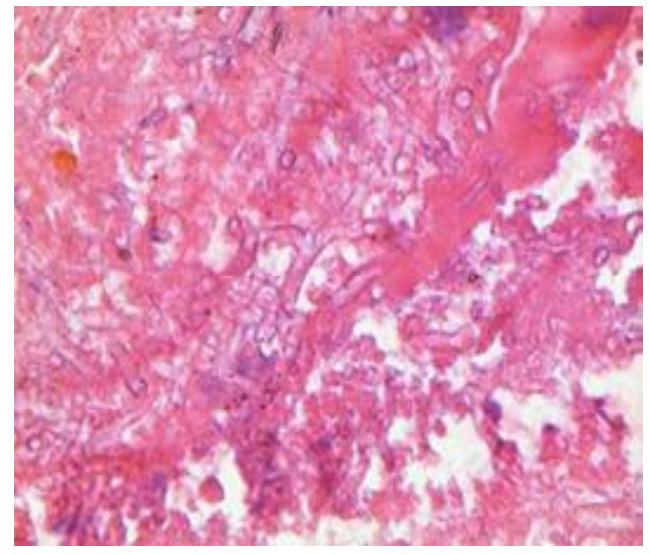

Fig 2: Lung sections showing broad ribbon like aseptate fungal hyphae with irregular branching. (H\&E staining 400x).

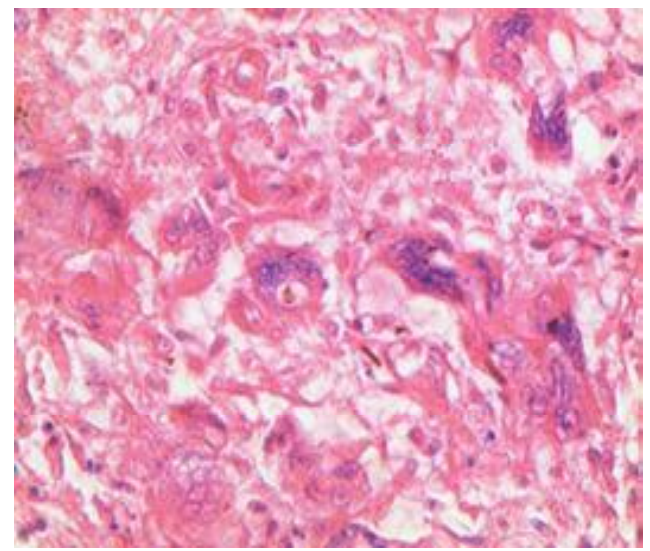

Fig 3: Lung sections: multi nuclaeatd giant cells with fungal hyphae (H\&E staining 400x).

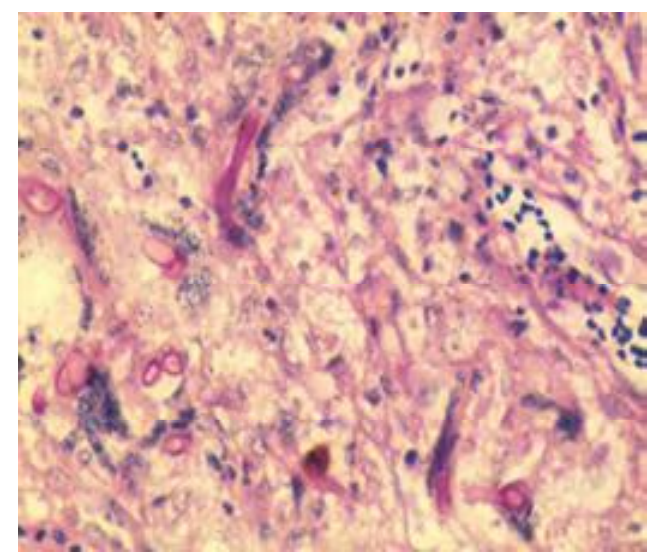

Fig 4: Lung sections: multi nuclaeatd giant cells with fungal hyphae (PAS staining 400x). characteristics of mycelia were in consistent with the hyphae of Zygomyces spp. Similar findings were described in mycotic pneumonia of sheep caused by zygomycetes sps in sheep (Pawaiya et al., 2015). The tissue sections of lungs revealed discrete and multiple necrotic foci, which sometimes covered wide diffuse areas due to coalescing, characterized by mixed type granulomatous inflammatory reaction. Mixed granulomatous lesions showed many eosinophilic as well as PAS-positive aseptate, broad, branched fungal hyphae, scattered throughout the pulmonary parenchyma.

\section{CONCLUSION}

In conclusion, basing on gross, histopathological lesions and morphological characteristics of fungal hyphae, it was diagnosed as Mycotic pneumonia with etiology of zygomycetes species.

\section{REFERENCES}

Chandler, F.W., Kaplan, E., Ajello, L. (1980). Histopathology of Mycotic Diseases. London. Wolfe Medical Publications, 122-127.

Chute, H.L. (1978). Fungal Infections. In: Diseases of Poultry. [Hofstad, Calnek, Helmboldt, Reid, Yoder, eds]. $7^{\text {th }}$ edn. lowa State University Press, Ames. 367.

Keyer, I.F. (1969). Mycoses. In: Diseases of Cage and Aviary Birds, [Petrak. eds]. Philadelphia. 453.

Migaki, G., Langheinrich, K.A., Garner, F.M. (1970). Pulmonary mucormycosis (Phycomycosis) in a chicken. Avian Diseases. 14: 179-183.

McCaskey, P.C., Langheinrich, K.A. (1984). Zygomycosis in the duck. Avian Diseases. 28: 791-798.

Pawaiya, R.V.S., Shivasharanappa, N., Sharma, N., Mishra, A.K., Gururaj, K., Paul, S., Gupta, V.K., Kumar. A., Sharma, D.K., Kumar, N., Singh, S. (2015). Pathomorphological study of a spontaneous case of Mycotic pneumonia in sheep. Indian Journal of Veterinary Pathology. 39(1): 78-80.

Perelman, B., Kuttin, E. (1992). Zygomycosis in ostriches. Avian Pathology. 21(4): 675-680.

Suzuta, F., Kimura, K., Urakawa. R., Kusuda, Y., Tanaka, S., Hanafusa, Y., Haritani, M. (2015). Variations in the morphology of Rhizomucor pusillus in granulomatous lesions of a Magellanic penguin (Spheniscus magellanicus). Journal of Veterinary Medical Science. 77(8): 1029-1031. 